\title{
Modulation of the renin-angiotensin system inhibits memory advantage for \\ negative emotional material via decreasing hippocampus activation and its
}

\section{coupling with the amygdala}

\section{Authors}

Ting Xu ${ }^{1}$, Xinqi Zhou ${ }^{1}$, Guojuan Jiao ${ }^{1}$, Yixu Zeng ${ }^{1}$, Weihua Zhao ${ }^{1}$, Jialin $\mathrm{Li}^{2}$, Fangwen $\mathrm{Yu}^{1}$, Feng Zhou ${ }^{1}$, Shuxia Yao ${ }^{1}$, Benjamin Becker ${ }^{1, *}$

\section{Affiliations}

${ }^{1}$ The Clinical Hospital of the Chengdu Brain Science Institute, MOE Key Laboratory for Neuroinformation, University of Electronic Science and Technology of China, Chengdu, China

${ }^{2}$ Max Planck School of Cognition, Leipzig, Germany

\section{*Correspondence to}

Benjamin Becker

University of Electronic Science and Technology

Xiyuan Avenue 2006, 611731 Chengdu, China

mail: ben_becker@gmx.de 


\section{Abstract}

Exaggerated arousal and dysregulated emotion-memory interactions are key pathological dysregulations that accompany the development of post-traumatic stress disorder (PTSD). Current treatments for PTSD are of moderate efficacy and preventing the dysregulations already during exposure to threatening events may attenuate the development of PTSD-symptomatology. The present proof-of-concept study examined the potential of a single dose of the angiotensin II type 1 receptor (AT1R) antagonist losartan (LT) to attenuate the mnemonic advantage of threatening stimuli and the underlying neural mechanism. In a preregistered double-blind, between-subject, placebo-controlled pharmaco-fMRI study we combined an emotional subsequent memory paradigm with $L T(n=29)$ or placebo treatment $(n=30)$ and a surprise memory test after 24 h washout. LT generally improved memory performance and abolished emotional memory enhancement for negative yet not positive material while emotional experience during encoding remained intact. LT further suppressed the hippocampus activity during encoding of subsequently remembered negative stimuli and abolished the positive association between higher activity in this region and subsequent better memory for negative material observed under placebo. On the network level LT reduced coupling between the hippocampus and the basolateral amygdala during successful encoding of negative stimuli. Overall, our findings suggest that LT has the potential to selectively attenuate memory formation for negative yet not positive information by decreasing hippocampus activity and its functional coupling strength with the left amygdala. These findings suggest a promising potential of $L T$ to prevent preferential encoding and remembering of negative events, a mechanism that could prevent the emotion-memory dysregulations underlying the development of PTSD-symptomatology.

Keywords: Angiotensin, PTSD, Trauma, Emotional memory, Hippocampus, Amygdala 


\section{Introduction}

Memory enhancement for threatening events and stimuli facilitates the avoidance of future dangers and thus represents an important evolutionary survival mechanism [1]. However dysregulation in this fine-grained interplay between emotion and memory may crucially contribute to the development of major psychiatric disorders [2], particularly post-traumatic stress disorder (PTSD) [3, 4]. PTSD is a prevalent and highly debilitating psychiatric disorder [5], which is characterized by hyperarousal and re-experiencing traumatic events frequently through disruptive memories and dissociative flashbacks [6]. Neurocognitive models of PTSD propose that symptoms manifesting as a consequence of trauma-induced dysregulations in emotion cognition interactions lead to biased encoding and subsequent memory for threat-associated information $[3,7,8]$. While maladaptive emotion-memory integration has been considered as a trans-diagnostic treatment target [9], the current interventions aim at suppressing exaggerated emotional responses or modifying the traumatic memory following the development of a psychiatric condition $[10,11]$. With respect to PTSD the available treatments are characterized by modest efficacy and high drop-out rates [12], and administering pharmacological agents immediately after trauma exposure did not result in the expected reduction of PTSD symptomatology $[13,14]$. As such a preventive pharmacological strategy which modulates encoding during the exposure to emotional events may represent an innovative and promising approach to prevent the development of PTSD symptoms.

Growing evidence from animal models and human findings have suggested a crucial role of the renin-angiotensin system (RAS) in emotion and memory formation which may overlap with processes relevant for the development of PTSD [15-17]. Different lines of research have indicated an important role of the RAS in the development of PTSD such that (1) blood levels of renin were elevated in individuals with trauma-experience [18], (2) treatment with angiotensin blockers, particularly the angiotensin II type 1 receptor antagonist losartan (LT) during trauma exposure reduced the incidence of PTSD $[19,20]$, and (3) administration of LT in healthy controls also reduced autonomic stress reactivity during experimental trauma induction [21] and shifted emotional reactivity from negative to positive information $[22,23]$. While these findings support that targeting the RAS via LT may prevent the development of PTSD by modulating emotional or memory formation processes, the underlying behavioral and neurobiological mechanisms have not been examined. 
Extensive research has implicated the amygdala and hippocampus in emotional memory formation, in the development of PTSD, and as potential target sites for the modulatory effects of LT on emotion and memory. The memory advantage of emotional over neutral material strongly depends on the interaction between the amygdala and hippocampus [1], with the amygdala mediating emotional arousal effects on the hippocampal formation which primarily encodes information into long term memory [24, 25]. Recent neuroimaging studies have demonstrated dysregulated hippocampal and amygdala processing during fear conditioning [26] and encoding of negative emotional stimuli [3], as well as regionally specific decreased volumes of the amygdala-hippocampal $[27,28]$ in PTSD patients. Treatment evaluation studies moreover have reported that functional dysregulations in amygdala and hippocampus normalize with decreasing PTSD symptomatology during successful interventions [29, 30], while these regions also mediated the modulatory effects of pharmacological agents on emotional memory formation [31]. The hippocampus and amygdala exhibit dense expressions of central angiotensin receptors that are considered to critically mediate learning-related neuroplasticity in these regions [32]. Animal models further support this by demonstrating that LT-induced enhanced general memory function and reduced contextual memories are mediated by effects on the hippocampus $[15,33]$, while effects on fear-related processes are primarily mediated by the amygdala [16, 34]. Initial pharmacological neuroimaging studies in humans also demonstrated LT-induced modulation of the amygdala and its connections with prefrontal regulatory regions during threat processing $[17,35]$. Together the animal and human studies may indicate a potential neural pathway which may mediate the attenuation of $L T$ administration to negative memory formation.

Against this background the present preregistered randomized double-blind placebo-controlled pharmaco-fMRI experiment sought to provide proof-of-concept for the treatment potential of LT in PTSD by examining the behavioral and neural effects of a single-dose of 50mg LT on emotional memory encoding and subsequent recognition. Following treatment administration participants were presented with negative, positive and neutral pictures during $\mathrm{fMRI}$ and underwent a surprise memory test after a washout period of 24 hours (similar approach see [36]). Based on previous findings suggesting an LT-induced facilitation of aversive memory extinction $[15,37,38]$, we expected that LT administration would particularly abolish the memory enhancement effects for negative stimuli. We further hypothesized these effects would be either mediated by decreased 
amygdala or hippocampal activity during encoding reflecting LT-induced attenuated emotional arousal or attenuated memory formation for negative materials, respectively. Given the critical role of interactions between the hippocampus and the amygdala for emotional memory formation, specifically the memory advantage of negative events, we expected that treatment-induced changes in regional activity would be mirrored in the functional interaction between these regions.

\section{Materials and Methods}

\section{Participants}

$\mathrm{N}=66$ heathy male participants were recruited for the present pre-registered randomized double-blind between-group pharmacological fMRI experiment. To account for variance related to sex differences in emotional memory related neural activity $[39,40]$ and the response to RA blockade [41] only male participants were enrolled in the present study. A total of 7 participants were excluded and lead to a final sample size of $n=59$ for further analysis ( $L T=29, P L C=30)$. For general enrollment criteria as well as exclusion criteria see Supplementary Materials: Participants and CONSORT flow diagram

The study procedures adhered to the latest reversion of the Declaration of Helsinki and were approved by the local ethics committee. All participants provided written informed consent for this study which was preregistered on Clinical Trials.gov (https://clinicaltrials.gov/ct2/show/NCT04606225).

\section{Study protocols}

A pharmacological fMRI experiment with a double-blind randomized, placebo-controlled, between-subject design was utilized to examine LT effects on encoding emotional information and subsequent memory performance. Prior to fMRI experiment participants were randomly assigned to administer either a single 50-mg p.o. dose of LT or PLC packed in identical capsules. An independent researcher not in direct contact with participants and experimenters dispensed the capsules according to a computer-generated randomization list. Psychometric assessments regarding mood, attention and memory capacity were administered at baseline and post experiment, whereas cardiovascular activity (i.e., blood pressure, heart rate) were measured at baseline, after drug 
administration and the experiment (Details see Supplementary Materials). Consistent with the pharmacodynamic profile of $\mathrm{LT}[42,43]$ the experiments started after peak plasma concentrations were reached (90 minutes after treatment). Participants first performed a reward processing task (Duration: 30 minutes) and next underwent the emotional memory paradigm during fMRI. Results of the reward processing task will be reported in a separate publication. After the experiments, participants were asked to guess whether they had received LT or PLC. 24 hours after the experiment a computerized surprise memory recognition test was implemented (Fig. 1a).

a

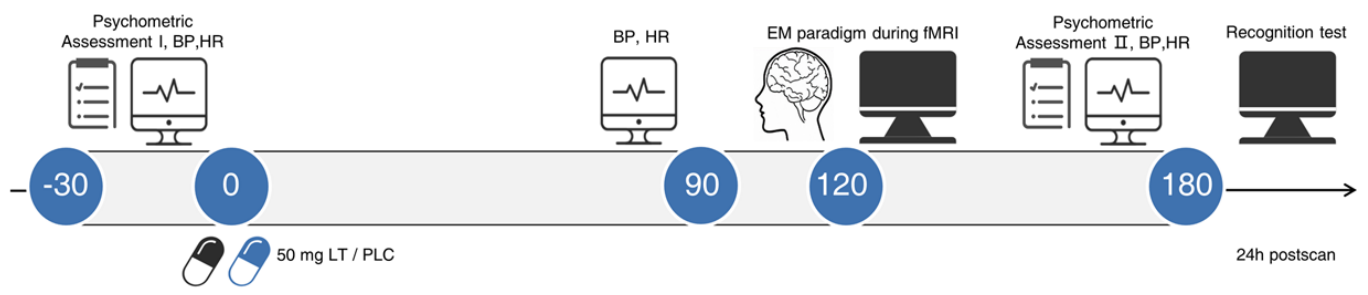

b

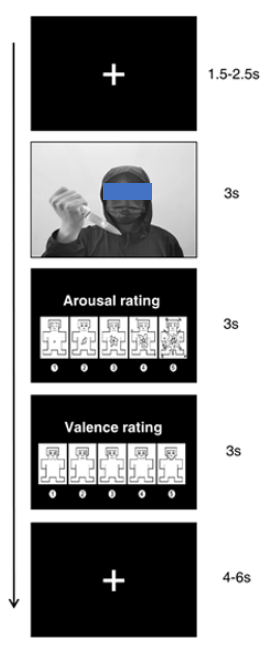

c

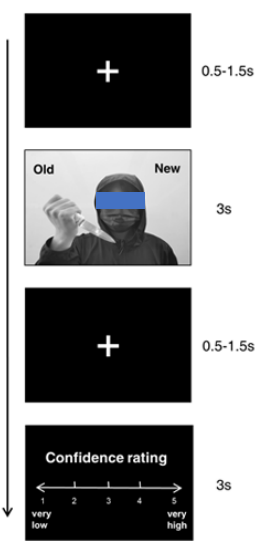

d
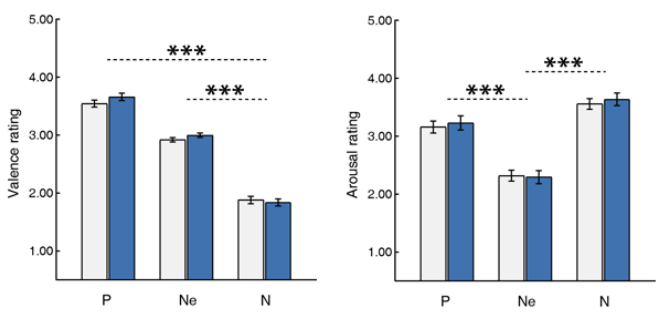

$\square$ LT

$\mathbf{e}$

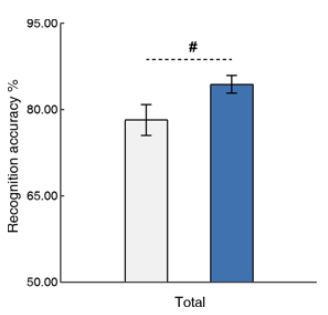

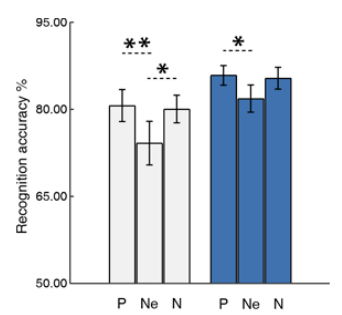

Fig. 1 Experimental timeline, experimental paradigm and treatment effects on emotional encoding and subsequent memory performance. a Experimental protocol, b - c Schematic representation and timing of the emotional memory and recognition paradigm. $\mathbf{d}$ Emotional characteristics of the paradigm, including valence-dependent effects on valence and arousal experience. e Treatment effects on memory performance, indicating that LT enhanced general memory whereas it abolished the memory-enhancing effects of negative emotional stimuli. BP, blood pressure; HR, heart rate; LT, losartan; PLC, placebo; EM, emotional memory paradigm; P, positive; N, negative; Ne, neutral; $\# p<0.08,{ }^{*} p<0.05,{ }^{* *} p<0.01, * * * p<0.001$. 


\section{Experimental paradigm}

The event-related fMRI emotional memory paradigm incorporated positive, neutral and negative pictorial stimuli from the Nencki Affective Picture System [44] and International Affective Picture System [45]. Three runs with 10 pictures per emotional condition were used and the order was balanced between treatment conditions (Stimuli details see Supplementary Materials). Each stimulus was presented for $3 \mathrm{~s}$ during encoding, followed by a jittered interval ranging from $1.5 \mathrm{~s}$ to $2.5 \mathrm{~s}$ and separate ratings for valence and arousal by means of the Self-Assessment Manikin scales (ranging from 1 (minimum) to 5 (maximum)) [46]. The rating scale was presented for $3 \mathrm{~s}$ and presentation order was balanced across participants. The trials were separated by a jittered inter-trial interval of 4-6s which served as low-level baseline.

24 hours after encoding participants completed a forced choice recognition memory test in the laboratory. The test included the 90 pictures previously shown during encoding intermixed with 54 novel images (18 per emotional condition, matched for valence and arousal; see Supplementary Materials). During the surprise memory test participants had to indicate whether the stimuli had been shown during encoding ('old' or 'new') and to rate their confidence for the response (Fig. 1b-c,

\section{Supplementary Materials).}

\section{Behavioral data analyses}

Statistical analyses were conducted in JASP (version 0.14.1.0). Effects of LT on emotional experience during encoding (valence, arousal) were analyzed by means of mixed ANOVAs with treatment (LT, PLC) as the between-subjects factor and valence (positive, negative, neutral) as the within-subjects factor. In line with previous studies $[47,48]$ recognition accuracy was computed by subtracting the false alarm rate for each emotional valence from its respective hit rate. Emotion-specific effects of LT on subsequent memory performance were next investigated by means of a mixed ANOVA with treatment (LT, PLC) as the between-subjects factor, valence (positive, negative, neutral) as the within-subjects factor and recognition accuracy as the dependent variable. In addition, separate mixed ANOVA models with identical between- and within-subjects factors were computed for false alarm and hit rate. The effects of LT on the emotional enhancement of memory were further explored by comparing recognition accuracy for each emotional condition with the neutral condition within each treatment group, the analysis which is consistent with previous work [49, 50]. This 
analysis was tested one sided, with $p<0.05$ due to the a priori hypothesis that LT may inhibit the memory-enhancing effects of negative stimuli.

\section{fMRI data acquisition, preprocessing and analysis}

MRI data were collected on a 3.0 Tesla system (GE MR750, General Electric Medical System, Milwaukee, WI, USA) and further preprocessed using standard procedures in SPM 12 (Statistical Parametric Mapping; http://www.fil.ion.ucl.ac.uk/spm/;Wellcome Trust Centre for Neuroimaging) (Details provided in Supplementary Materials). We modelled two separate event-related general linear models (GLM) on the first level to separately examine effects of LT on emotional experience during encoding and subsequent memory formation.

The first GLM model examined effects of treatment on emotional experience and modelled presentation of each stimulus according to the valence condition (positive, negative, neutral) using an event-related design and convolution with the hemodynamic response function [51]. Rating periods (arousal, valence) and six head motion parameters were included as covariates. In line with our a priori regional hypotheses the group level analysis focused on the amygdala and hippocampus as defined by Jülich-Düsseldorf brain (JuBrain) cytoarchitectonic atlas based structural masks [52, 53]. The extracted estimates from hippocampus and amygdala were subjected to two separate mixed ANOVA models with treatment as the between-subject factor and valence as the within-subject factor. The second GLM aimed at determining effects of treatment on emotional memory formation by modelling the difference in memory ( $\mathrm{Dm}$ ) effect by differentiating the valence-specific subsequently remembered from subsequently forgotten items [54]. On the group level effects of treatment were examined via extraction of parameter estimates from atlas-based amygdala-hippocampal regions and a subsequent analysis of task-modulated functional connectivity to determine treatment effects on hippocampal-amygdala interactions. All analyses incorporated adequate correction for multiple comparisons, on the voxel-level Family-Wise Error (FWE) correction adopted to the size of the ROI and a threshold value of $p<0.05$ was employed (details see Supplementary Materials).

\section{Results}




\section{Sociodemographic, psychometric and physiological characteristics}

The LT ( $n=29)$ and PLC $(n=30)$ groups exhibited comparable sociodemographic and psychometric properties (Table 1 ; all ps $>0.13$ ). Previous studies suggested that the effects of $L T$ on cardiovascular activity was only observed after at least 3 hours while the central effects occurred in a faster manner $[55,56]$. Consistent with this, 80 minutes after the LT application examination of blood pressure and heart rate revealed no significant treatment difference (all ps>0.11, Supplementary Materials, Table S1).

Table 1. Sociodemographic and Psychometric assessments

\begin{tabular}{|c|c|c|c|c|}
\hline & Time & $\operatorname{PLC}(n=29)$ & $\operatorname{LT}(n=30)$ & $t$ test, $p$ value \\
\hline Age, years & & $20.83 \pm 2.05$ & $20.48 \pm 2.98$ & 0.52 \\
\hline Body Mass Index, kg/m2 & & $21.19 \pm 2.17$ & $21.28 \pm 2.01$ & 0.86 \\
\hline \multirow{2}{*}{ PANAS - positive } & Baseline & $26.80 \pm 5.89$ & $27.38 \pm 4.86$ & 0.68 \\
\hline & Post experiment & $25.87 \pm 6.19$ & $24.69 \pm 5.44$ & 0.44 \\
\hline \multirow{2}{*}{ PANAS - negative } & Baseline & $15.07 \pm 5.12$ & $17.03 \pm 7.48$ & 0.24 \\
\hline & Post experiment & $13.17 \pm 5.08$ & $14.45 \pm 6.60$ & 0.41 \\
\hline \multirow{2}{*}{ STAI - state } & Baseline & $39.77 \pm 8.86$ & $42.28 \pm 10.32$ & 0.32 \\
\hline & Post experiment & $38.17 \pm 7.78$ & $40.41 \pm 9.46$ & 0.32 \\
\hline \multirow{2}{*}{ STAI - trait } & Baseline & $41.13 \pm 7.19$ & $41.90 \pm 9.85$ & 0.73 \\
\hline & Post experiment & $40.27 \pm 7.81$ & $41.59 \pm 8.83$ & 0.55 \\
\hline \multirow{2}{*}{ BDI II } & Baseline & $8.43 \pm 8.97$ & $9.17 \pm 7.07$ & 0.73 \\
\hline & Post experiment & $6.17 \pm 7.31$ & $7.34 \pm 6.01$ & 0.50 \\
\hline \multirow{2}{*}{ DCAT1 } & Baseline & $38.13 \pm 5.71$ & $38.17 \pm 6.56$ & 0.98 \\
\hline & Post experiment & $56.17 \pm 9.06$ & $55.55 \pm 8.02$ & 0.78 \\
\hline \multirow{2}{*}{ DCAT2 } & Baseline & $65.10 \pm 12.91$ & $64.17 \pm 10.59$ & 0.76 \\
\hline & Post experiment & $47.07 \pm 6.24$ & $45.28 \pm 6.52$ & 0.29 \\
\hline \multirow{2}{*}{ DCAT3 } & Baseline & $61.50 \pm 9.76$ & $58.72 \pm 8.33$ & 0.25 \\
\hline & Post experiment & $70.50 \pm 12.56$ & $68.55 \pm 9.15$ & 0.50 \\
\hline \multirow{2}{*}{ Working memory } & Baseline & $0.86 \pm 0.09$ & $0.86 \pm 0.09$ & 0.87 \\
\hline & Post experiment & $0.91 \pm 0.08$ & $0.87 \pm 0.10$ & 0.14 \\
\hline
\end{tabular}


Values are presented as mean \pm SD. PLC, placebo; LT, Losartan; PANAS, Positive and Negative Affect Schedule; STAI, Spielberger State-Trait Anxiety Inventory; BDI II, Beck Depression Inventory II; DCAT 1-3, Digit cancellation test 1-3.

\section{Effects of LT on emotional experience during encoding}

The mixed ANOVA with treatment (LT, PLC) as the between-subjects factor, valence (positive, negative, neutral) as the within-subjects factor, and valence or arousal ratings as dependent variable revealed a significant main effects of valence (valence, Greenhouse-Geisser, $F_{(1.43,57)}=542.02, p<0.001$, partial $\eta^{2}=0.91 ;$ arousal, $F_{(2,57)}=227.06, p<0.001$, partial $\eta^{2}=0.80$, Fig. $\left.1 d\right)$, but no significant main or interaction effect involving treatment (all ps $>0.29$ ). The neutral pictures were rated less positive than positive ones $\left(t_{(58)}=12.01, p<0.001, d=1.56\right)$, and were rated less negative than negative ones $\left(t_{(58)}=20.54, p<0.001, d=2.68\right)$. The negative pictures were perceived as more arousing than positive $\left(t_{(58)}=6.50, p<0.001, d=0.85\right)$ and neutral pictures $\left(t_{(58)}=20.83, p<0.001, d=2.71\right)$ irrespective of treatment.

\section{Effects of LT on subsequent emotional memory performance}

Examining $L T$ effects on subsequent recognition accuracy by means of a treatment $(L T, P L C) \times$ valence (positive, negative, neutral) mixed ANOVA revealed a significant main effect of valence (Greenhouse-Geisser, $F_{(1.77,57)}=6.12, p<0.01$, partial $\eta^{2}=0.10$ ) and a marginally significant main effect of treatment $\left(F_{(1,57)}=3.77, p=0.06\right.$, partial $\left.\eta^{2}=0.06\right)$, but no significant interaction effect $(p>0.66)$. Post-hoc tests revealed an enhanced recognition performance for emotional relative to neural pictures in both groups (positive vs. neutral, $\mathrm{t}_{(58)}=3.18, \mathrm{p}=0.01, \mathrm{~d}=0.41$; negative vs. neutral, $\mathrm{t}_{(58)}=2.85$, $p=0.01, d=0.37)$, and a general improved memory performance across valence following LT administration $\left(t_{(57)}=1.94, p=0.06, d=0.25\right.$, Fig. 1e). For the hit and false alarm rates, the treatment main effect was only observed for hit $\left(F_{(1,57)}=4.25, p=0.04\right.$, partial $\left.\eta^{2}=0.07\right)$ but not false alarm rates suggesting that the effect was driven by better recognition of items presented under LT (further details see Supplementary Materials).

To test our hypothesis that LT may specifically attenuate memory performance for negative events we directly examined the emotional memory enhancement within each treatment group. In the PLC group recognition memory for negative $\left(t_{(29)}=2.10, p=0.05, d=0.38\right)$ and positive pictures $\left(t_{(29)}=2.81\right.$, $p=0.01, d=0.51$ ) was significantly enhanced relative to neutral ones, whereas the LT group exhibited a 
memory-enhancing effects only for positive stimuli $\left(t_{(28)}=2.34, p=0.03, d=0.43\right.$, Fig. 1e) yet not for negative stimuli $\left(t_{(28)}=1.35, p=0.19, d=0.25\right.$, Fig. 1e).

\section{Effects of LT on neural activity during emotion encoding}

Examination of extracted parameter estimates from the amygdala revealed a significant main effect of valence $\left(F_{(2,57)}=9.53, p<0.001\right.$, partial $\eta^{2}=0.14$, Fig. 2a), but no significant main or interaction effect of treatment (all ps>0.12). The post-hoc tests indicated higher amygdala activity during encoding of negative pictures relative to neutral $\left(t_{(58)}=4.28, p<0.001, d=0.56\right)$ and positive ones $\left(t_{(58)}=2.90, p<0.01, d=0.38\right)$ in both treatment groups. In contrast a significant valence $\times$ treatment interaction effect $\left(F_{(2,57)}=3.69, p=0.03\right.$, partial $\left.\eta^{2}=0.06\right)$ was observed in the hippocampus, whereas no main effects of valence or treatment (all ps $>0.14$ ) were observed in this region. Post-hoc tests revealed that following LT hippocampus activity was significantly decreased during encoding for negative stimuli compared to both positive $\left(t_{(28)}=-2.96, p<0.01, d=-0.55\right.$, Fig. 2 b) and neutral ones $\left(t_{(28)}=-2.43, p=0.02, d=-0.45\right)$.

a
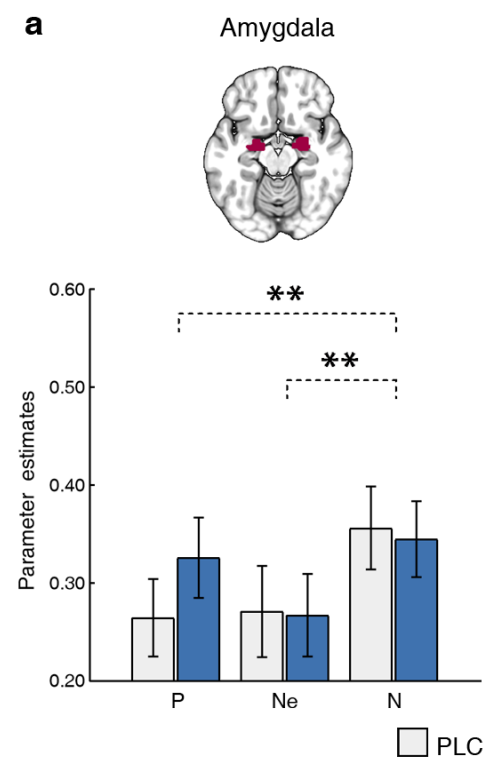

b
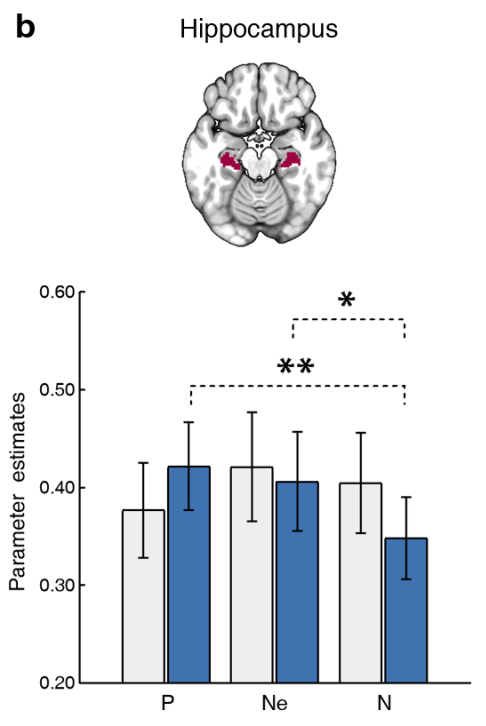

$\square$ LT

Fig. 2 Examination of LT effects on neural activation during emotion encoding. a The amygdala showed increased activity during encoding for negative stimuli relative to positive and neutral ones in both treatment groups. b Following LT hippocampus activity decreased significantly when encoding negative stimuli compared to the positive and neutral ones whereas there was no difference for hippocampus response in PLC group. Error bars represent standard error of the mean. $\mathrm{P}$, positive; Ne, neutral; $\mathrm{N}$, negative; PLC, placebo; LT, losartan; ${ }^{*} \mathrm{p}<0.05,{ }^{*} \mathrm{p}<0.01$. 


\section{Effects of LT on neural activity during subsequent emotional memory formation}

In general the successful emotional memory formation was associated with increased responses in the bilateral amygdala (left, peak MNI, $-20 /-2 /-28, \mathrm{~T}_{(53)}=4.84, \mathrm{P}_{\text {svc-FwEpeak }}=0.002, \mathrm{k}=180$; right, peak $\mathrm{MNI}, 22 /-2 /-28, \mathrm{~T}_{(53)}=4.05, \mathrm{P}_{\text {svc-FWEpeak }}=0.02, \mathrm{k}=119$, Fig. S2) and the right hippocampus (peak MNI, $30 /-28 /-16, T_{(53)}=4.24, P_{\text {SVC-FWEpeak }}=0.01, k=21$, Fig. S2).

In a next step effects of treatment on the identified amygdala-hippocampal nodes involved in successful memory formation were examined by means of extraction of parameter estimates from independently defined atlas-based regions for the amygdala and hippocampus. Examination of the amygdala revealed a significant main effect of valence (left, $F_{(1,52)}=19.49, p<0.001$, partial $\eta^{2}=0.27$; right, $F_{(1,52)}=10.62, p=0.002$, partial $\left.\eta^{2}=0.17\right)$, but no main or interaction effect involving treatment (all ps $>0.22$ ). Post-hoc tests demonstrated increased amygdala activity for subsequent memory formation for negative pictures relative to positive ones (left, $t_{(54)}=4.42, p<0.001, d=0.60$; right, $t_{(54)}=3.26, p=0.002, d=0.44$, Fig. 3a-b). The right hippocampus only exhibited a marginal valence $x$ treatment effect $\left(F_{(1,52)}=3.33, p=0.07\right.$, partial $\left.\eta^{2}=0.06\right)$. Specifically, this region displayed comparable activity during subsequent memory formation for positive and negative stimuli during PLC (t $(26)=-0.30, p=0.77, d=-0.06$ ), while during $L T$ decreased activity for negative memory formation compared with positive memory formation $\left(t_{(26)}=-2.49, p=0.02, d=-0.48\right.$, Fig. $\left.3 c\right)$. Further examination revealed that right hippocampal activity was positively associated with recognition accuracy for negative pictures following PLC ( $r h o=0.28, p=0.08$, one sided) but this association was absent following LT administration ( $r$ ho $=-0.25, p=0.11$, significant correlation differences between groups, Fisher's $z=1.88, p=0.06$, Fig. $3 d$ ). 


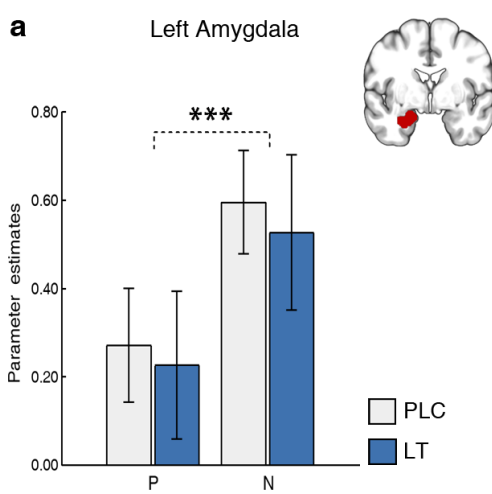

c

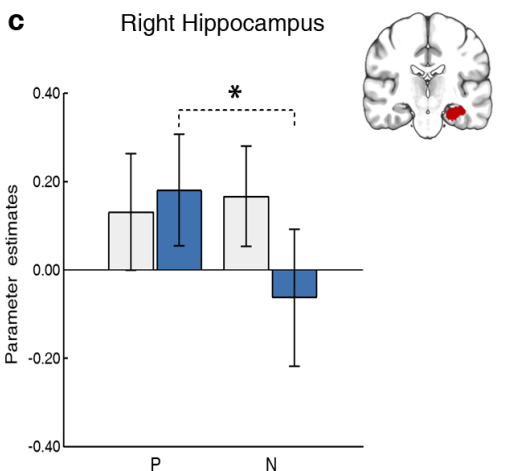

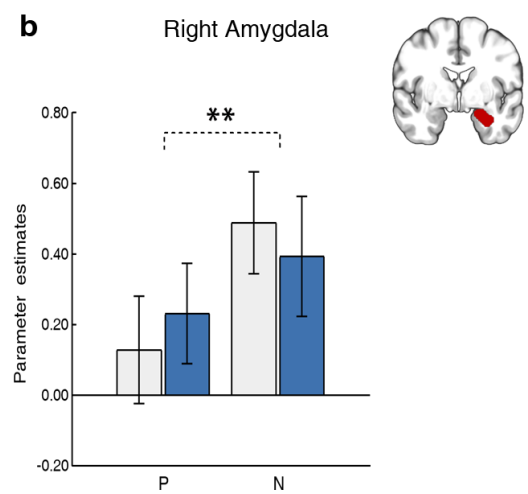

d Right Hippocampus and negative memory

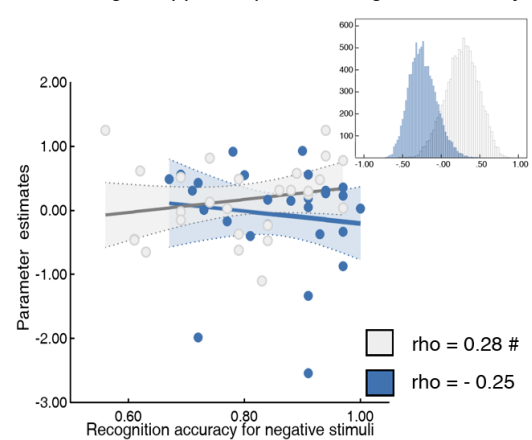

Fig. 3 LT effects on neural activity during successful memory formation for emotional stimuli. a-b Bilateral amygdala displayed increased activation during memory formation for negative stimuli relative to positive ones irrespective of treatment. c Right hippocampus activity decreased during memory formation for negative stimuli following LT administration while comparable hippocampus activation was observed for successful encoding of positive and negative stimuli following PLC. $\mathbf{d}$ Memory performance for negative stimuli was positively related to right hippocampus activity following PLC but not LT treatment. The histograms represent the bootstrapped distribution of correlation coefficients (10 000 iterations). Error bars represent standard error of the mean. $\mathrm{P}$, positive; N, negative; PLC, placebo; LT, losartan; $\# p<0.08$, one sided, ${ }^{*} p<0.05,{ }^{*} p<0.01,{ }^{* * *} p<0.001$.

\section{Effects of LT on hippocampal-amygdala coupling}

Given the importance of hippocampus-amygdala interaction for emotional memory formation a functional connectivity analysis was conducted to examine treatment effects on the coupling between these regions. During successful emotional memory formation a significant valence $x$ treatment interaction effect was found on right hippocampus coupling with the left amygdala (peak MNI, $-32 /-6 /-20, P_{\text {svc-FwEpeak }}=0.021, k=8, F_{(1,52)}=14.87$, Fig. 4). Probabilistic mapping demonstrated that the identified cluster was located in the basolateral amygdala subregion (78.1\% probability). Extracting parameter estimates from the left amygdala revealed that LT significantly decreased right 
hippocampus coupling strength with left amygdala during successful memory for negative stimuli relative to $P L C\left(t_{(52)}=-2.04, p<0.05, d=-0.55\right)$.
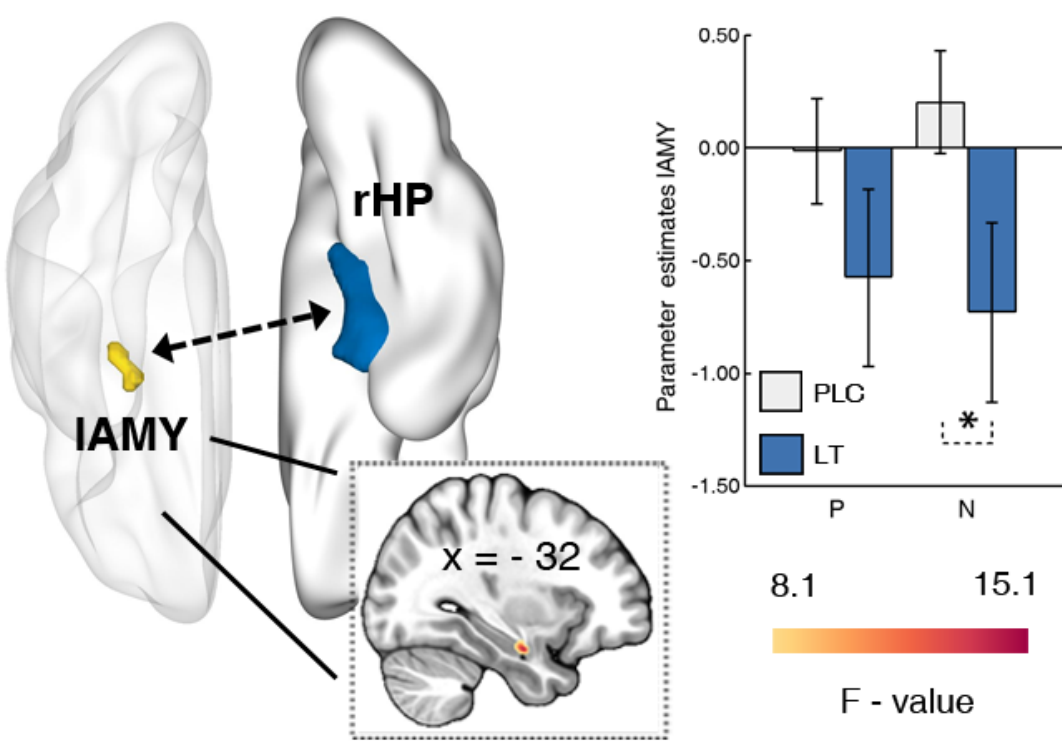

Fig. 4 LT effects on right hippocampus connectivity with the left amygdala during successful emotional memory formation. LT specifically decreased coupling strength of the right hippocampus with the left basolateral amygdala during memory formation of subsequently successfully remembered negative stimuli. Error bars represent standard error of the mean. IAMY, left amygdala; rHP, right hippocampus; P, positive; N, negative; PLC, placebo; LT, losartan. ${ }^{*} p<0.05$.

\section{Discussion}

The present study examined whether pharmacological modulation of the RAS via LT during emotional encoding can attenuate the subsequent memory advantage of negative material and whether this effect is mediated by effects on the amygdala or hippocampus. In line with our prediction, LT slightly enhanced general memory performance while it inhibited the memory advantage for negative information in the absence of modulatory effects on emotional arousal or valence. The abolished mnemonic enhancement for negative material was paralleled by decreased hippocampus activity during encoding of negative stimuli, while amygdala activation was not affected. Exploring brain-behavior associations revealed that following PLC better subsequent memory performance for negative stimuli was associated with higher right hippocampus activity during encoding, while this association was abolished following LT. On the network level LT specifically decreased the functional communication of the right hippocampus with the left basolateral amygdala (BLA) during successful memory for negative stimuli. Together these findings 
suggest an important contribution of the RAS to memory formation for negative stimuli via valence-specific modulation of hippocampus activation and its coupling with the BLA. In contrast, emotional evaluation and associated amygdala activity during encoding remained unaffected by RAS blockade.

Despite converging evidence emphasizing the role of hyperarousal and a negative memory encoding bias during trauma exposure for the development of PTSD, the underlying mechanisms have been difficult to target therapeutically $[3,7,8]$. Previous observational studies demonstrated that blockade of the RAS via LT during trauma exposure reduced the incidence of PTSD and hypothesized that the beneficial effects may be mediated by an LT-induced attenuation of autonomic reactivity or arousal $[19,20]$. In the present study LT did not affect subjective arousal experience while it slightly enhanced memory performance and abolished the memory advantage of negative material. Our findings suggest that LT may attenuate preferential memory formation for negative stimuli via an indirect arousal-mediated mechanism but rather a valence-specific reduction in the memory advantage of negative emotional material. This functional profile of LT could point to a therapeutic mechanism with the potential to attenuate exaggerated emotional memory formation for negative materials, a mechanism which may attenuate the risk for memory dysregulations that facilitate the development of PTSD.

Neuroimaging studies in PTSD commonly linked symptoms in the domains of hyperarousal and emotional memory to dysregulations in the amygdala-hippocampal complex. Some evidence suggests that hyperarousal and emotional-reactivity symptoms in PTSD are strongly mediated by amygdalar dysregulations $[57,58]$ while memory dysfunctions are associated with hippocampal dysregulations [59]. However, during threat learning and encoding of negative emotional materials both regions have been found to be dysregulated in $\operatorname{PTSD}[26,50,60,61]$. The present study demonstrated that LT specifically decreased hippocampus activity during encoding of negative stimuli while not affecting amygdala functioning. This finding may explain the behavioral effects of an LT-induced abolishment of memory advantage of negative material in the absence of effects on arousal. Moreover animal models have demonstrated an important role of the hippocampus in mediating effects of LT on aversive memory, such that (1) an LT-induced reduction in fear memories triggered by interoceptive cues was mediated by attenuated cFOS expression in the hippocampus [33], and (2) the preference for safe relative to threat environments was mediated by an LT-induced 
suppression of hippocampal AT1R [62]. The engagement of the hippocampus in negative memory formation and its modulation via LT was further supported by the observation of a positive association between hippocampal activity and memory performance for negative material following PLC which was abolished by LT.

In addition to the hippocampus, the amygdala plays a critical role in emotion-memory integration [1] as well as emotional memory dysregulations in PTSD [50]. The amygdala is considered a key locus for enhanced responses to emotionally arousing [25], particularly threatening stimuli [63] and consequently modulates the arousal-mediated enhancement of emotional memories. Although a previous study reported an LT-induced prolonged amygdala threat reactivity in humans [35], the present study did not observe direct effects on amygdala activity yet a valence-specific attenuated functional coupling between the right hippocampus and the contralateral BLA during negative memory formation. Whereas LT may not have affected arousal or emotional processing associated amygdala activity per se, it suppressed communication between the amygdala and the hippocampus - a pathway that has been strongly involved in encoding, consolidation and reconsolidation for negative and threatening materials across species $[64,65]$.

Previous studies also explored pharmacological strategies to attenuate emotional memory formation, e.g. by means of the $\beta$-adrenergic blocker propranolol, the NMDA receptor antagonist ketamine, or 3,4-Methylenedioxymethamphetamine. However, the effects were commonly mediated by biased emotion-associated amygdala reactivity [31], impaired general memory performance [49], as well as abolished emotional enhancement for both positive and negative material [66]. In contrast, LT (1) specifically abolished the advantage of negative material, while not impairing general or positive memory performance (e.g. NMDA antagonists), and (2) did not affect general valence or arousal processing which may lead to biased reactions or decision making. Together, the pattern of observed behavioral and neural effects of LT may point to a promising mechanism to prevent exaggerated negative emotional memories already during the exposure stage while preserving general memory formation and emotional processing.

Finally, our results have to be interpreted in the context of limitations. First while the present study reduced variance related to gender difference future works should confirm the generalization of the observed effects to female individuals. Second, given the relatively short retention interval of 24h longer term effects of LT on emotional memory and consolidation need to be determined. 
Further, emotional stimuli can initiate complex interactions between hormonal systems that are coordinated by the hypothalamic-pituitary-adrenal axis (HPA) encompassing central and peripheral sites [67]. Previous animal models also suggest a contribution of both central and peripheral AT1R to HPA mediated emotional processes [68]. As such the potential contribution of effects on peripheral AT1R to the central effects of LT needs to be explored in future studies.

In conclusion, the present study provides the first evidence for a role of the RAS in valence-specific memory formation via modulating the hippocampus and its connections with the BLA. RAS blockade via $\mathrm{LT}$ abolished the memory advantage for negative events in the absence of effects on emotional processing. On the neural level this attenuation was mirrored in valence-specific decreased hippocampus activity and coupling of this region with the BLA. Together these findings provide proof-of-concept for the functional profile of $L T$ to inhibit dysregulated negative memory formation, a mechanism which have the potential to prevent the development of trauma-induced emotional memory dysregulations.

\section{Acknowledgements and disclosure}

This work was supported by the National Key Research and Development Program of China (2018YFA0701400). Data availiability: unthresholded group-level statistical maps are available on OSF (https://osf.io/v4j9d/). Other data of this study is available from the corresponding author upon reasonable request. The authors report no biomedical financial interests or potential conflicts of interest.

\section{Author contributions}

TX and BB designed the study; TX, GJ, YZ, XZ conducted the experiment and collected the data. TX, $\mathrm{XZ}, \mathrm{FZ}, \mathrm{FY}, \mathrm{L}$ performed the data analysis. TX and BB wrote the manuscript draft, WZ and YS critically revised the manuscript draft. 


\section{References}

1. Talmi, D. (2013). Enhanced emotional memory: Cognitive and neural mechanisms. Current Directions in Psychological Science, 22(6), 430-436.

2. Mitte, K. (2008). Memory bias for threatening information in anxiety and anxiety disorders: a meta-analytic review. Psychological Bulletin, 134(6), 886.

3. Durand, F., Isaac, C., \& Januel, D. (2019). Emotional memory in post-traumatic stress disorder: A systematic PRISMA review of controlled studies. Frontiers in psychology, 10, 303.

4. Woud, M. L., Verwoerd, J., \& Krans, J. (2017). Modification of cognitive biases related to posttraumatic stress: A systematic review and research agenda. Clinical psychology review, 54, 81-95.

5. Kessler, R. C., Aguilar-Gaxiola, S., Alonso, J., Benjet, C., Bromet, E. J., Cardoso, G., . . Ferry, F. (2017). Trauma and PTSD in the WHO world mental health surveys. European journal of psychotraumatology, 8(sup5), 1353383.

6. Edition, F. (2013). Diagnostic and statistical manual of mental disorders. Am Psychiatric Assoc, 21.

7. Chemtob, C., Roitblat, H., Hamada, R. S., Carlson, J. G., \& Twentyman, C. T. (1988). A cognitive action theory of post-traumatic stress disorder. Journal of anxiety disorders, 2(3), 253-275.

8. Hayes, J. P., VanElzakker, M. B., \& Shin, L. M. (2012). Emotion and cognition interactions in PTSD: a review of neurocognitive and neuroimaging studies. Frontiers in integrative neuroscience, 6, 89.

9. Kindt, M., \& van Emmerik, A. (2016). New avenues for treating emotional memory disorders: towards a reconsolidation intervention for posttraumatic stress disorder. Therapeutic advances in psychopharmacology, 6(4), 283-295.

10. Myers, K. M., \& Davis, M. (2007). Mechanisms of fear extinction. Molecular psychiatry, 12(2), 120-150.

11. Orsini, C. A., \& Maren, S. (2012). Neural and cellular mechanisms of fear and extinction memory formation. Neuroscience \& Biobehavioral Reviews, 36(7), 1773-1802.

12. Carpenter, J. K., Andrews, L. A., Witcraft, S. M., Powers, M. B., Smits, J. A., \& Hofmann, S. G. (2018). Cognitive behavioral therapy for anxiety and related disorders: A meta-analysis of randomized placebo-controlled trials. Depression and anxiety, 35(6), 502-514.

13. Sijbrandij, M., Kleiboer, A., Bisson, J. I., Barbui, C., \& Cuijpers, P. (2015). Pharmacological prevention of post-traumatic stress disorder and acute stress disorder: a systematic review and meta-analysis. The Lancet Psychiatry, 2(5), 413-421.

14. Wright, L. A., Sijbrandij, M., Sinnerton, R., Lewis, C., Roberts, N. P., \& Bisson, J. I. (2019). Pharmacological prevention and early treatment of post-traumatic stress disorder and acute stress disorder: a systematic review and meta-analysis. Translational psychiatry, 9(1), 1-10.

15. Bild, W., Hritcu, L., Stefanescu, C., \& Ciobica, A. (2013). Inhibition of central angiotensin II enhances memory function and reduces oxidative stress status in rat hippocampus. Progress in Neuro-Psychopharmacology and Biological Psychiatry, 43, 79-88.

16. Marvar, P. J., Goodman, J., Fuchs, S., Choi, D. C., Banerjee, S., \& Ressler, K. J. (2014). Angiotensin type 1 receptor inhibition enhances the extinction of fear memory. Biological psychiatry, 75(11), 864-872.

17. Zhou, F., Geng, Y., Xin, F., Li, J., Feng, P., Liu, C., .. . Ebstein, R. P. (2019). Human extinction learning is accelerated by an angiotensin antagonist via ventromedial prefrontal cortex and its connections with basolateral amygdala. Biological psychiatry, 86(12), 910-920.

18. Terock, J., Hannemann, A., Janowitz, D., Freyberger, H. J., Felix, S. B., Dörr, M., . . Grabe, H. J. (2019). Associations of trauma exposure and post-traumatic stress disorder with the activity of the 
renin-angiotensin-aldosterone-system in the general population. Psychological medicine, 49(5), 843-851.

19. Khoury, N. M., Marvar, P. J., Gillespie, C. F., Wingo, A., Schwartz, A., Bradley, B., . . Ressler, K. J. (2012).

The renin-angiotensin pathway in posttraumatic stress disorder: angiotensin-converting enzyme inhibitors and angiotensin receptor blockers are associated with fewer traumatic stress symptoms. The Journal of clinical psychiatry, 73(6), 0-0.

20. Seligowski, A. V., Duffy, L. A., Merker, J. B., Michopoulos, V., Gillespie, C. F., Marvar, P. J., ... Ressler, K. J. (2021). The renin-angiotensin system in PTSD: a replication and extension. Neuropsychopharmacology, 46(4), 750-755.

21. Shkreli, L., Woud, M. L., Ramsbottom, R., Rupietta, A. E., Waldhauser, G. T., Kumsta, R., \& Reinecke, A. (2020). Angiotensin involvement in trauma processing-exploring candidate neurocognitive mechanisms of preventing post-traumatic stress symptoms. Neuropsychopharmacology, 45(3), 507-514.

22. Pulcu, E., Shkreli, L., Holst, C. G., Woud, M. L., Craske, M. G., Browning, M., \& Reinecke, A. (2019). The effects of the angiotensin II receptor antagonist losartan on appetitive versus aversive learning: a randomized controlled trial. Biological psychiatry, 86(5), 397-404.

23. Zhou, X., Xu, T., Zeng, Y., Zhang, R., Qi, Z., Zhao, W., .. Becker, B. (2021). The angiotensin antagonist Losartan shifts social reward motivation and punishment feedback sensitivity via modulating midbrain-striato-frontal circuits. bioRxiv.

24. Desmedt, A., Marighetto, A., Richter-Levin, G., \& Calandreau, L. (2015). Adaptive emotional memory: the key hippocampal-amygdalar interaction. Stress, 18(3), 297-308.

25. Richardson, M. P., Strange, B. A., \& Dolan, R. J. (2004). Encoding of emotional memories depends on amygdala and hippocampus and their interactions. Nature neuroscience, 7(3), 278-285.

26. Suarez-Jimenez, B., Albajes-Eizagirre, A., Lazarov, A., Zhu, X., Harrison, B. J., Radua, J., ... Fullana, M. A. (2020). Neural signatures of conditioning, extinction learning, and extinction recall in posttraumatic stress disorder: a meta-analysis of functional magnetic resonance imaging studies. Psychological medicine, 50(9), 1442-1451.

27. Logue, M. W., van Rooij, S. J., Dennis, E. L., Davis, S. L., Hayes, J. P., Stevens, J. S., . . Koch, S. B. (2018). Smaller hippocampal volume in posttraumatic stress disorder: a multisite ENIGMA-PGC study: subcortical volumetry results from posttraumatic stress disorder consortia. Biological psychiatry, 83(3), 244-253.

28. Nelson, M. D., \& Tumpap, A. M. (2017). Posttraumatic stress disorder symptom severity is associated with left hippocampal volume reduction: a meta-analytic study. CNS spectrums, 22(4), 363-372.

29. Garrett, A., Cohen, J. A., Zack, S., Carrion, V., Jo, B., Blader, J., . . Agras, W. S. (2019). Longitudinal changes in brain function associated with symptom improvement in youth with PTSD. Journal of psychiatric research, 114, 161-169.

30. Zhu, X., Suarez-Jimenez, B., Lazarov, A., Helpman, L., Papini, S., Lowell, A., . . Schneier, F. (2018). Exposure-based therapy changes amygdala and hippocampus resting-state functional connectivity in patients with posttraumatic stress disorder. Depression and anxiety, 35(10), 974-984.

31. Strange, B., \& Dolan, R. J. (2004). $\beta$-Adrenergic modulation of emotional memory-evoked human amygdala and hippocampal responses. Proceedings of the National Academy of Sciences, 101(31), 11454-11458.

32. Wright, J. W., Reichert, J. R., Davis, C. J., \& Harding, J. W. (2002). Neural plasticity and the brain renin-angiotensin system. Neuroscience \& Biobehavioral Reviews, 26(5), 529-552. 
33. Winter, A., Ahlbrand, R., \& Sah, R. (2019). Recruitment of central angiotensin II type 1 receptor associated neurocircuits in carbon dioxide associated fear. Progress in Neuro-Psychopharmacology and Biological Psychiatry, 92, 378-386.

34. Yu, Z., Swiercz, A. P., Moshfegh, C. M., Hopkins, L., Wiaderkiewicz, J., Speth, R. C., . . Marvar, P. J. (2019). Angiotensin II type 2 receptor-expressing neurons in the central amygdala influence fear-related behavior. Biological psychiatry, 86(12), 899-909.

35. Reinecke, A., Browning, M., Breteler, J. K., Kappelmann, N., Ressler, K. J., Harmer, C. J., \& Craske, M. G. (2018). Angiotensin regulation of amygdala response to threat in high-trait-anxiety individuals. Biological Psychiatry: Cognitive Neuroscience and Neuroimaging, 3(10), 826-835.

36. Striepens, N., Scheele, D., Kendrick, K. M., Becker, B., Schäfer, L., Schwalba, K., . . Hurlemann, R. (2012). Oxytocin facilitates protective responses to aversive social stimuli in males. Proceedings of the National Academy of Sciences, 109(44), 18144-18149.

37. do Nascimento Lazaroni, T. L., Bastos, C. P., Moraes, M. F. D., Santos, R. S., \& Pereira, G. S. (2016). Angiotensin-(1-7)/Mas axis modulates fear memory and extinction in mice. Neurobiology of learning and memory, 127, 27-33.

38. Parrish, J. N., Bertholomey, M. L., Pang, H. W., Speth, R. C., \& Torregrossa, M. M. (2019). Estradiol modulation of the renin-angiotensin system and the regulation of fear extinction. Translational psychiatry, 9(1), 1-12.

39. Cahill, L., Haier, R. J., White, N. S., Fallon, J., Kilpatrick, L., Lawrence, C., . . Alkire, M. T. (2001). Sex-related difference in amygdala activity during emotionally influenced memory storage. Neurobiology of learning and memory, 75(1), 1-9.

40. Canli, T., Desmond, J. E., Zhao, Z., \& Gabrieli, J. D. (2002). Sex differences in the neural basis of emotional memories. Proceedings of the National Academy of Sciences, 99(16), 10789-10794.

41. Miller, J. A., Cherney, D. Z., Duncan, J. A., Lai, V., Burns, K. D., Kennedy, C. R., . . Scholey, J. W. (2006). Gender differences in the renal response to renin-angiotensin system blockade. Journal of the American Society of Nephrology, 17(9), 2554-2560.

42. Getyala, A., V Gangadharappa, H., Sarat Chandra Prasad, M., Praveen Kumar Reddy, M., \& M Pramod Kumar, T. (2013). Formulation and evaluation of non-effervescent floating tablets of losartan potassium. Current drug delivery, 10(5), 620-629.

43. Sica, D. A., Gehr, T. W., \& Ghosh, S. (2005). Clinical pharmacokinetics of losartan. Clinical pharmacokinetics, 44(8), 797-814.

44. Marchewka, A., Żurawski, Ł., Jednoróg, K., \& Grabowska, A. (2014). The Nencki Affective Picture System (NAPS): Introduction to a novel, standardized, wide-range, high-quality, realistic picture database. Behavior research methods, 46(2), 596-610.

45. Lang, P. J., Bradley, M. M., \& Cuthbert, B. N. (1997). International affective picture system (IAPS): Technical manual and affective ratings. NIMH Center for the Study of Emotion and Attention, 1(39-58), 3.

46. Bradley, M. M., \& Lang, P. J. (1994). Measuring emotion: the self-assessment manikin and the semantic differential. Journal of behavior therapy and experimental psychiatry, 25(1), 49-59.

47. Adelman, J. S., \& Estes, Z. (2013). Emotion and memory: A recognition advantage for positive and negative words independent of arousal. Cognition, 129(3), 530-535.

48. Mickley Steinmetz, K. R., Schmidt, K., Zucker, H. R., \& Kensinger, E. A. (2012). The effect of emotional arousal and retention delay on subsequent-memory effects. Cognitive neuroscience, 3(3-4), 150-159. 
49. Becker, B., Steffens, M., Zhao, Z., Kendrick, K. M., Neumann, C., Weber, B., ... Hurlemann, R. (2017). General and emotion-specific neural effects of ketamine during emotional memory formation. Neuroimage, 150, 308-317.

50. Brohawn, K. H., Offringa, R., Pfaff, D. L., Hughes, K. C., \& Shin, L. M. (2010). The neural correlates of emotional memory in posttraumatic stress disorder. Biological psychiatry, 68(11), 1023-1030.

51. Friston, K. J., Holmes, A. P., Worsley, K. J., Poline, J. P., Frith, C. D., \& Frackowiak, R. S. (1994). Statistical parametric maps in functional imaging: a general linear approach. Human brain mapping, 2(4), 189-210.

52. Amunts, K., Kedo, O., Kindler, M., Pieperhoff, P., Mohlberg, H., Shah, N., . . Zilles, K. (2005). Cytoarchitectonic mapping of the human amygdala, hippocampal region and entorhinal cortex: intersubject variability and probability maps. Anatomy and embryology, 210(5-6), 343-352.

53. Amunts, K., \& Zilles, K. (2015). Architectonic mapping of the human brain beyond Brodmann. Neuron, 88(6), 1086-1107.

54. Paller, K. A., \& Wagner, A. D. (2002). Observing the transformation of experience into memory. Trends in cognitive sciences, 6(2), 93-102.

55. Goldberg, M. R., Tanaka, W., Barchowsky, A., Bradstreet, T. E., McCrea, J., Lo, M.-W., ... Bjornsson, T. D. (1993). Effects of losartan on blood pressure, plasma renin activity, and angiotensin II in volunteers. Hypertension, 21(5), 704-713.

56. Mechaeil, R., Gard, P., Jackson, A., \& Rusted, J. (2011). Cognitive enhancement following acute losartan in normotensive young adults. Psychopharmacology, 217(1), 51-60.

57. Ben-Zion, Z., Shany, O., Admon, R., Keynan, N. J., Avisdris, N., Balter, S. R., . . Hendler, T. (2021). Differential roles of positive and negative valence systems in the development of post-traumatic stress psychopathology. bioRxiv.

58. Etkin, A., \& Wager, T. D. (2007). Functional neuroimaging of anxiety: a meta-analysis of emotional processing in PTSD, social anxiety disorder, and specific phobia. American journal of Psychiatry, 164(10), 1476-1488.

59. Carletto, S., Panero, M., Cavallo, M., \& Pagani, M. (2021). Neurobiology of Posttraumatic Stress Disorder PET and SPECT in Psychiatry (pp. 411-435): Springer.

60. Dickie, E. W., Brunet, A., Akerib, V., \& Armony, J. L. (2008). An fMRI investigation of memory encoding in PTSD: influence of symptom severity. Neuropsychologia, 46(5), 1522-1531.

61. Hayes, J. P., LaBar, K. S., McCarthy, G., Selgrade, E., Nasser, J., Dolcos, F., \& Morey, R. A. (2011). Reduced hippocampal and amygdala activity predicts memory distortions for trauma reminders in combat-related PTSD. Journal of psychiatric research, 45(5), 660-669.

62. Tashev, R., \& Ivanova, M. (2018). Involvement of hippocampal angiotensin 1 receptors in anxiety-like behaviour of olfactory bulbectomized rats. Pharmacological Reports, 70(5), 847-852.

63. Becker, B., Mihov, Y., Scheele, D., Kendrick, K. M., Feinstein, J. S., Matusch, A., . . Oros-Peusquens, A.-M. (2012). Fear processing and social networking in the absence of a functional amygdala. Biological psychiatry, 72(1), 70-77.

64. de Voogd, L. D., Klumpers, F., Fernández, G., \& Hermans, E. J. (2017). Intrinsic functional connectivity between amygdala and hippocampus during rest predicts enhanced memory under stress. Psychoneuroendocrinology, 75, 192-202.

65. Hermans, E. J., Kanen, J. W., Tambini, A., Fernández, G., Davachi, L., \& Phelps, E. A. (2017). Persistence of amygdala-hippocampal connectivity and multi-voxel correlation structures during awake rest after fear learning predicts long-term expression of fear. Cerebral Cortex, 27(5), 3028-3041. 
66. Doss, M. K., Weafer, J., Gallo, D. A., \& de Wit, H. (2018). MDMA impairs both the encoding and retrieval of emotional recollections. Neuropsychopharmacology, 43(4), 791-800.

67. LaBar, K. S., \& Cabeza, R. (2006). Cognitive neuroscience of emotional memory. Nature Reviews Neuroscience, 7(1), 54-64.

68. Armando, I., Carranza, A., Nishimura, Y., Hoe, K.-L., Barontini, M., Terrón, J. A., . . Saavedra, J. M. (2001). Peripheral administration of an angiotensin II AT1 receptor antagonist decreases the hypothalamic-pituitary-adrenal response to isolation stress. Endocrinology, 142(9), 3880-3889. 